\title{
Efficient Reconstruction of Band-Limited Sequences from Nonuniformly Decimated Versions by Use of Polyphase Filter Banks
}

\author{
P. P. VAIDYANATHAN, SENior Member, IEeE, AND VINCENT C. LIU, MEMber, IEeE
}

\begin{abstract}
Based on recent results on the reconstruction of a bandlimited sequence from a nonuniformly decimated version, this paper develops an efficient polyphase structure for such reconstruction. Theoretically, the reconstruction involves the implementation of a bank of multilevel filters, and this paper shows how all these reconstruction filters can be obtained at the cost of one $M$ th band low-pass filter and a constant matrix multiplier. The resulting structure is therefore more general than the ad hoc scheme reported recently. In addition, the method offers a direct means of controlling the overall reconstruction distortion $T(z)$ by appropriate design of a low-pass prototype filter $P(z)$. Extension of these results to multiband band-limited signals and to the case of nonconsecutive nonuniform subsampling are also summarized, along with generalizations to the multidimensional case. Design examples are included to demonstrate the theory, and the complexity of the new method is seen to be much lower than earlier ones.
\end{abstract}

\section{INTRODUCTION}

$\mathrm{T}$ HE theory of nonuniform sampling, and techniques for reconstruction of a signal from unevenly spaced samples have a long history dating back to the fundamental paper by Shannon [1]. Subsequent papers by Papoulis [2] and Brown [3] throw more light on the topic from a linear-system viewpoint. An excellent review on this topic is given in [4].

In a recent article [5, sec. IV], the authors of this paper have interpreted the reconstruction of a band-limited sequence from a nonuniformly decimated version, in terms of maximally decimated filter-bank structures [6]-[10]. Before outlining the purpose of the present paper, it is necessary to first summarize the main result of this interpretation. Suppose $x(n)$ is a low-pass sequence which is band limited to the region $0 \leq|\omega|<L \pi / M$ where $L$ and $M$ are integers with $L<M$. It is then possible to perform a nonuniform decimation of $x(n)$ without losing information, as follows: divide the time axis $n$ into segments of length $M$, and retain only $L$ consecutive samples in each segment. Thus, the retained samples are $x(n M-$ $k), 0 \leq k \leq L-1$. The signal $x(n)$ can be reconstructed from these samples by using a set of $L$ linear time-in-

Manuscript received November 7, 1988; revised December 1, 1989. This work was supported in part by National Science Foundation grants DCI 8552579 and MIP 8604456.

P. P. Vaidyanathan is with the Department of Electrical Engineering, California Institute of Technology, Pasadena, CA 91125.

V. C. Liu was with the Department of Electrical Engineering. California Institute of Technology. Pasadena, CA 91125. He is now with the VideoCipher Division of the General Instruments Corporation. San Diego, CA 92121 .

IEEE Log Number 9038419.

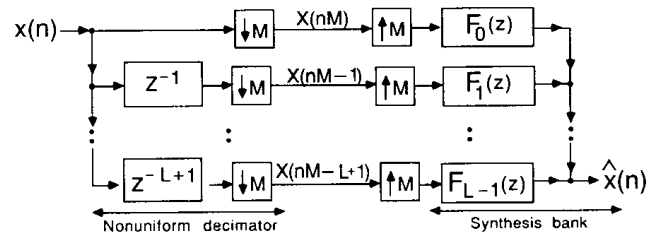

Fig. 1. The nonuniform decimation and reconstruction scheme.

variant filters $F_{k}(z), 0 \leq k \leq L-1$ ( collectively called a synthesis filter bank $\left.\left\{F_{k}(z)\right\}\right)$. The nonuniform decimation and reconstruction can be represented as in Fig. 1 where the symbols $\downarrow M$ and $\uparrow M$ denote $M$-fold decimators and interpolators, respectively, as defined in [6]-[10]. In [5], a closed form expression for the synthesis filters was derived for arbitrary $L, M$. The implementation aspects of the synthesis bank $\left\{F_{k}(z)\right\}$ were not addressed, even though design examples for the special cases of $L=2, M$ $=3$ and $L=3, M=4$ were presented. The synthesis filer-bank structures for these special cases were obtained in a somewhat ad hoc manner, without noticing or exploiting the special properties of these filters to reduce complexity. Moreover, even though the polyphase approach [11] has been used to advantage in other sections of [5] for other reasons, it was not used in [5, sec. IV], because the advantage in this specific context was not evident at that time.

A formal mathematical solution for the filters $\left\{F_{k}(z)\right\}$ [5, eq. (56)] reveals that each $z^{-k} F_{k}(z)$ is a multilevel filter, i.e., $e^{-j \omega k} F_{k}\left(e^{j \omega}\right)$ is a (complex) piecewise constant function of $\omega$ (more precise statement will be given in Section II). We shall use this in Section II of this paper to show that all the $L$ filters $\left\{F_{k}(z)\right\}$ can be implemented by 1) implementing a single low-pass prototype filter in terms of its $M$ polyphase components, and 2) forming linear combinations of the outputs of these polyphase components. As a result, the reconstruction cost is equal to the cost of a single low-pass filter (plus the overhead of implementing the constant linear combiner, which, as we shall see, is typically only a small fraction). In Section III we consider the $L=2, M=3$ case in order to demonstrate the theory. We include a detailed design example, and include complexity comparisons with [5]. More wellknown techniques for fractional decimation and reconstruction (such as the ones in [6, ch. 2]) are also included 
in this comparison. The results of this paper easily extend to the case when the $L$ samples retained per segment are nonconsecutive, and also to the case when the signal $x(n)$ is multiband rather than low-pass, with total spectral occupancy of $2 \pi L / M$. These extensions, and extensions to the multidimensional case are summarized in Sections IV and $\mathrm{V}$.

\section{A. Notations}

Throughout the paper the integers $L$ and $M$ have the significance described above. Boldface type denotes matrices and vectors. The row and column indices begin at zero. Matrices are often indicated in terms of their entries as $\boldsymbol{A}=\left[A_{k m}\right]$ where $k$ is the row index and $m$ is the column index. The quantities $\boldsymbol{A}^{*}, \boldsymbol{A}^{T}$, and $\boldsymbol{A}^{\dagger}$ denote, respectively, the conjugate, transpose, and conjugate transpose of $\boldsymbol{A}$. We denote $W_{M}=e^{-j 2 \pi / M}$, and if the subscript is omitted it is taken to be $M$. Any transfer function $P(z)$ can be written in the form [11] $P(z)=\sum_{l=0}^{M-1} z^{-1} P_{l}\left(z^{M}\right)$ which is called the type 1 polyphase decomposition. $P_{l}(z)$ are unique for fixed $P(z)$ and $M$, and are called the type 1 polyphase components.

I) Special Matrices: The $M \times M$ DFT matrix is $W$ $=\left[W^{k m}\right]$. The $L \times L$ leading principal submatrix of $\boldsymbol{W}$ is denoted as $\boldsymbol{V}$, i.e., the $(k, m)$ entry of $\boldsymbol{V}$ is given by $V_{k m}$ $=W^{k m}, 0 \leq k, m \leq L-1$. Note that $V$ is Vandermonde with distinct columns so that it is nonsingular. We define $\boldsymbol{U}=\boldsymbol{V}^{-1}$ with the columns of $\boldsymbol{U}$ denoted as $\boldsymbol{u}_{i}$, so that $\boldsymbol{U}$ $=\left[\boldsymbol{u}_{0} \boldsymbol{u}_{1} \cdots \boldsymbol{u}_{L-1}\right]$. The $M \times L$ upper left submatrix of $\boldsymbol{W}$ is denoted $\boldsymbol{R}$, i.e., $\boldsymbol{R}_{k m}=W^{k m}, 0 \leq k \leq M-1,0 \leq$ $m \leq L-1$. We define $J$ to be the permutation of the identity matrix with 1 's on the cross diagonal. A $3 \times 3$ example is

$$
\boldsymbol{J}=\left[\begin{array}{lll}
0 & 0 & 1 \\
0 & 1 & 0 \\
1 & 0 & 0
\end{array}\right]
$$

The $i$ th unit vector $[0 \cdots 010 \cdots 0]^{T}$ with " 1 " in the $i$ th position is denoted as $\boldsymbol{e}_{i}$. Finally, the diagonal matrices $\boldsymbol{D}_{i}$ and $\boldsymbol{\Lambda}$ with dimensions $L \times L$ and $M \times M$ are defined as

$$
\begin{aligned}
D_{i} & =\operatorname{diag}\left[1, W^{i}, \cdots, W^{(L-1) i}\right] \\
\mathbf{\Lambda} & =\operatorname{diag}\left[1, W^{(L-1) / 2}, \cdots, W^{(M-1)(L-1) / 2}\right] .
\end{aligned}
$$

\section{Polyphase Implementation of the} Reconstruction Filter Bank $\left\{F_{k}(z)\right\}$

Consider Fig. 1 where $x(n)$ is a low-pass signal band limited to $0 \leq|\omega|<L \pi / M$. Assume without loss of generality that $L$ and $M$ are relatively prime. The set of $L$ branches labeled as "nonuniform decimator" retains the samples $x(n M-k), 0 \leq k \leq L-1$. The decimators and interpolators create aliasing and imaging effects [6], respectively. As a result, the signal $\hat{X}(z)$ is a linear combination of $X(z)$ as well as the alias components $X\left(z W^{-1}\right)$, i.e.,

$$
\hat{X}(z)=\sum_{l=0}^{M-1} A_{l}(z) X\left(z W^{-1}\right)
$$

where [10]

$$
A_{l}(z)=\frac{1}{M} \sum_{k=0}^{L-1} z^{-k} W^{k l} F_{k}(z), \quad 0 \leq l \leq M-1 .
$$

If the filters $\left\{F_{k}(z)\right\}$ are chosen to cancel the aliasing terms (i.e., force $A_{l}(z)=0, l>0$ ), then we have $\hat{X}(z)=T(z) X(z)$. Here

$$
T(z) \triangleq A_{0}(z)=\frac{1}{M} \sum_{k=0}^{L-1} z^{-k} F_{k}(z)
$$

is called the distortion function of the system. The set of synthesis filters which results in perfect reconstruction (i.e., $\hat{x}(n)=x(n)$ ) is given by the following theorem [5].

Theorem 1: With reference to Fig. 1 let

$$
f(z)=\frac{1}{M}\left[F_{0}(z) z^{-1} F_{1}(z) \cdots z^{-\{L-1)} F_{L-1}(z)\right]^{T} .
$$

Then the set of filters $\left\{F_{k}(z)\right\}$ which results in perfect recovery (i.e., $\hat{x}(n)=x(n)$ ) for all inputs $x(n)$ band limited to $|\omega|<L \pi / M$ satisfies

$f\left(e^{j \omega}\right)= \begin{cases}\boldsymbol{D}_{i} \boldsymbol{u}_{i} & \omega \in \text { Region } i, 0 \leq i \leq L-1 \\ 0 & \text { otherwise }\end{cases}$

where Region $i$ corresponds to $-L \pi / M+2 \pi i / M<\omega$ $<-L \pi / M+2 \pi(i+1) / M$.

The $L$ regions are shown in Fig. 2 for clarity. From (6) we conclude that the filter $z^{-k} F_{k}(z)$ has a constant frequency response over the Region $i$. These constant levels $\beta_{k i}$ are typically complex, and depend upon both the filter number $k$ and the region number $i$. Filters with responses of this form are called multilevel filters. They are evidently ideal filters ${ }^{1}$ requiring infinite order (we shall approximate these with practical filters later). We shall show in Section II-A that any such filter can be realized as a linear combination $\sum_{n=0}^{M-1} b_{l} z^{-l} P_{l}\left(z^{M}\right)$ where $P_{l}(z)$ are the type 1 polyphase components of an ideal low-pass filter with response as in Fig. 3(a), i.e.,

$$
P\left(e^{j \omega}\right)= \begin{cases}1 & \text { for } 0 \leq|\omega|<\frac{L \pi}{M} \\ 0 & \text { otherwise. }\end{cases}
$$

So the $L$ filters $\left\{F_{k}(z)\right\}$ can be realized at the cost of one low-pass filter (plus the cost of linear combinations which is $M L-L^{2}$ real multipliers (Section II-B)) .

\section{A. Polyphase Structure for a Multilevel Filter}

Let $H(z)$ denote the transfer function of a multilevel filter with frequency response as in Fig. 4 (where $\beta_{i}^{\prime} s$ can

'For ideal filters, even though the response can be defined graphically and $F_{k}\left(e^{j \omega}\right)$ written down, the $z$ transform may not converge in the usual sense. We continue to use the notation $F_{k}(z)$ for convenience, with the understanding that $z$ is an abbreviation for $e^{j \omega}$. 


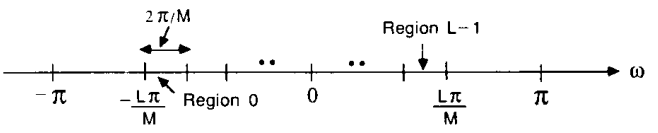

Fig. 2. The $L$ regions in $-\pi \leq \omega<\pi$.

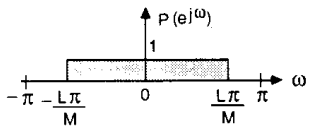

(a)

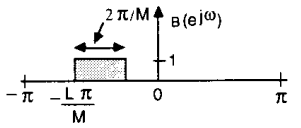

(b)
Fig. 3. (a) The ideal low-pass prototype and (b) ideal bandpass prototype.

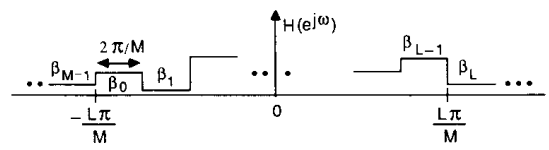

Fig. 4. A multilevel response. Here $\beta_{\text {; }}$ can in general be complex.

be complex ). The vector $\boldsymbol{b} \triangleq\left[\beta_{0} \beta_{1} \cdots \beta_{M-1}\right]^{T}$ will be called the level vector for the filter $H(z)$. It is clear that we can generate such a response by repeated use of shifted versions of an ideal bandpass response $B\left(e^{j \omega}\right)$ shown in Fig. 3(b). We shall, however, find it more convenient to obtain $H(z)$ starting from the prototype low-pass response $P\left(e^{j \omega}\right)$, for reasons mentioned in paragraph following Theorem 4; our immediate aim here is to prove the following result which relates the low-pass response $P\left(e^{j \omega}\right)$ to the multilevel response $H\left(e^{j \omega}\right)$.

Theorem 2: Let the low-pass prototype $P(z)$ be expressed in type 1 polyphase form, $P(z)=\sum_{l=0}^{M-1}$ $z^{-l} P_{l}\left(z^{M}\right)$. Then the multilevel filter $H(z)$ with levels $\beta_{i}$ as shown in Fig. 4 can be obtained as

$$
H(z)=\sum_{l=0}^{M-1} \frac{\alpha_{l}}{q_{l}} z^{-l} P_{l}\left(z^{M}\right)
$$

where

$$
q_{l} \triangleq \sum_{k=0}^{L-1} W^{-k l}, \quad 0 \leq l \leq M-1
$$

and the numbers $\alpha_{l}$ are related to the levels $\beta_{i}$ by $\alpha_{l}=$ $\sum_{i=0}^{M-1} \beta_{i} W^{-l i}$.

Remark: Notice that the quantity $q_{l}$ can be simplified as

$q_{l}=\gamma_{l} W^{-(L-1) l / 2}, \quad$ with

$\gamma_{l} \triangleq \sin (\pi L l / M) / \sin (\pi l / M), \quad 0 \leq l \leq M-1$.

Since $L$ and $M$ are relatively prime, one can verify that $\gamma_{1}$ $\neq 0$ for $0 \leq l \leq M-1$. Pictorially, the theorem says that the multilevel filter can be implemented as in Fig. 5 where $g^{T}(z)$ is an $M$-input one-output system defined by

$$
\begin{aligned}
& \boldsymbol{g}^{T}(z)=\left[G_{0}\left(z^{M}\right) z^{-1} G_{1}\left(z^{M}\right) \cdots z^{-(M-1)} G_{M-1}\left(z^{M}\right)\right] \\
& G_{l}(z) \triangleq P_{l}(z) / \gamma_{l} .
\end{aligned}
$$

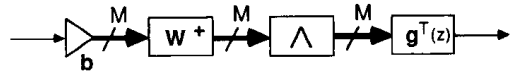

Fig. 5. Implementation of the multilevel filter $H(z)$. Here $b$ is the level vector.

Note that the quantities $G_{l}(z)$ have real coefficients since the ideal filter $P(z)$ (and hence $P_{l}(z)$ ) have real coefficients. In practice, one replaces $P(z)$ with an approximation, and using (8) one obtains an approximation of the multilevel filter. The "levels"' are adjustable by changing the multipliers $\beta_{i}$ (components of $\boldsymbol{b}$ in Fig. 5) without redesigning $G_{l}(z)$.

Proof of Theorem 2: From Fig. 3 it is clear that $P(z)$ $=\sum_{k=0}^{L-1} B\left(z W^{k}\right)$. By writing $B(z)$ in polyphase form i.e., as

$$
B(z)=\sum_{l=0}^{M-1} z^{-l} B_{l}\left(z^{M}\right)
$$

and substituting into $P(z)$, we obtain $P(z)=\sum_{l=0}^{M-1}$ $q_{l} z^{-1} B_{l}\left(z^{M}\right)$ so that

$$
P_{l}(z)=q_{l} B_{l}(z) .
$$

Similarly, we can express the multilevel filter $H(z)$ as $H(z)=\sum_{k=0}^{M-1} \beta_{k} B\left(z W^{k}\right)$ which simplifies to $H(z)=$ $\Sigma_{l=0}^{M-1} \alpha_{l} z^{-l} B_{l}\left(z^{M}\right)$. This reduces to (8) in view of (14).

\section{B. Implementation of the Multilevel Synthesis Bank $\left\{F_{k}(z)\right\}$}

From Fig. 5 we can mathematically express $H(z)$ as $H(z)=\boldsymbol{g}^{T}(z) \boldsymbol{\Lambda} \boldsymbol{W}^{\dagger} \boldsymbol{b}$. Using Theorem 2 each of the components $z^{-k} F_{k}(z) / M$ in (5) can be implemented as in Fig. 5. From (6), the level-vector for $z^{-k} F_{k}(z) / M$ will be

$$
\begin{aligned}
& \boldsymbol{b}_{k}=\left[\begin{array}{lllllll}
u_{k, 0} & W^{k} u_{k, 1} & \cdots & W^{k(L-1)} u_{k, L-1} & 0 & \cdots & 0
\end{array}\right]^{T} \\
& 0 \leq k \leq L-1
\end{aligned}
$$

which takes the place of $b$ in Fig. 5. Defining the $M \times L$ matrix $B=\left[b_{0} b_{1} \cdots b_{L-1}\right]$ we can express $f(z)$ as

$$
\boldsymbol{f}^{T}(z)=\boldsymbol{g}^{T}(z) C
$$

where $\boldsymbol{C} \triangleq \boldsymbol{\Lambda} \boldsymbol{W}^{\dagger} \boldsymbol{B}$ is $M \times L$. In view of the zero-valued entries in (15), we can rewrite

$$
C=\Lambda R^{*} A
$$

where $\boldsymbol{A}$ is the upper $L \times L$ submatrix of $\boldsymbol{B}$, i.e.,

$$
\boldsymbol{A}=\left[\begin{array}{lllll}
\boldsymbol{D}_{0} \boldsymbol{u}_{0} & \boldsymbol{D}_{1} \boldsymbol{u}_{1} & \cdots & \boldsymbol{D}_{L-1} \boldsymbol{u}_{L-1}
\end{array}\right]^{T} .
$$

In terms of these notations, we can implement the synthesis bank $\left\{F_{k}(z)\right\}$ as in Fig. 6. Note that the advance operators $z^{k}$ can be avoided in practice by inserting an overall delay $z^{-(L-1)}$ in front of all the $L$ inputs to $C$. Summarizing, we have proved:

Theorem 3: Let $x(n)$ in Fig. 1 be a low-pass signal band limited to $|\omega|<L \pi / M$. Then $x(n)$ can be recovered from the nonuniformly sampled version (i.e., from the set of samples $x(n M-k), 0 \leq k \leq L-1)$ as follows: design a low-pass filter $P(z)$ with response as in Fig. 3(a). With $P_{l}(z)$ denoting the type 1 polyphase com- 


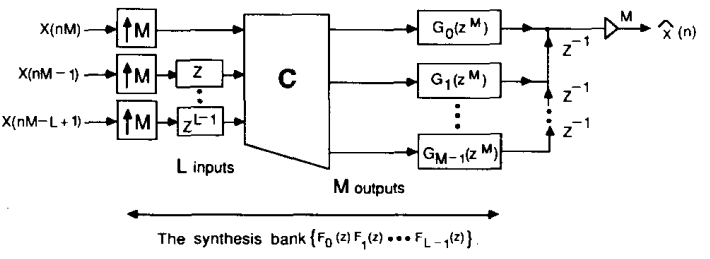

Fig. 6. The complete multilevel synthesis bank, implemented in terms of $G_{1}(z)$ and $C$.

ponents of this filter, define $G_{i}(z)$ as in (12). Compute the matrix $C$ from (16b). Then $\hat{x}(n)=x(n)$ (i.e., we have perfect recovery from the nonuniformly decimated signal) if $f(z)$ defined in (5) satisfies (16a).

In practice one can replace $P(z)$ with an FIR filter and compute the FIR components $G_{l}(z)$ in Fig. 6 using (12). The quantities $\boldsymbol{A}, \boldsymbol{R}, \boldsymbol{\Lambda}$ are not affected by this choice. The cost of the reconstruction system is equal to that of implementing $P(z)$ and the matrix $C$. Some useful features of the matrix $C$ are stated next.

Lemma 1: The $M \times L$ matrix $C$ has the following properties: 1) All the entries of the 0th row are equal to unity; 2) $C_{i k}=0$, for $i \leq k \leq L-1,1 \leq i \leq L-1$; 3) $C_{i k}$ $=0$, for $0 \leq k \leq i-M+L-1, M-L+1 \leq i \leq$ $M-1$; and 4) all entries are real valued.

To physically visualize the meaning of this, we display $C$ for $M=7, L=4$ below:

$$
C=\left[\begin{array}{llll}
1 & 1 & 1 & 1 \\
x & 0 & 0 & 0 \\
x & x & 0 & 0 \\
x & x & x & 0 \\
0 & x & x & x \\
0 & 0 & x & x \\
0 & 0 & 0 & x
\end{array}\right]
$$

where $x$ denotes arbitrary real numbers. There are two "triangles" of zeros in this matrix. These are induced by properties 2 and 3 of the Lemma. Because of Property 4 , the approximations $F_{k}(z)$ have real-valued impulse responses (as long as the approximation $P(z)$ has real coefficients). So $\hat{x}(n)$ is real for real $x(n)$, a desirable result. According to the lemma there are $L^{2}$ trivial entries ( 0 's and 1's) so that at most $M L-L^{2}$ real multipliers are involved in implementing $C$. A proof of the Lemma is included in the Appendix.

\section{Practical Implementation of the Reconstruction Scheme}

If $P(z)$ has the ideal response of Fig. 3(a) then aliasing is completely eliminated (i.e., $A_{l}(z)=0, l \neq 0$ ) and the distortion function $T(z)$ defined in (4) is $T(z)=1$ so that $\hat{x}(n)=x(n)$. We now consider the effects of replacing $P(z)$ with a nonideal filter. Since the ideal $P(z)$ has real coefficients, we restrict the approximant to have real coefficients. Evidently, aliasing is only approximately cancelled, and $\hat{x}(n)$ is only approximately band limited. Apart from these obvious effects, $\hat{X}(z)$ differs from $X(z)$ for another reason, viz., the distortion function $T(z)$ is not unity. Thus, even after the aliasing terms have been satisfactorily reduced, $T(z)$ introduces amplitude and phase distortions. The following important result tells us what the value of $T(z)$ will actually be.

Theorem 4: Consider the system of Fig. 1 where the synthesis bank is implemented as in Fig. 6, with $G_{l}(z)$ as in (12) where $P_{l}(z)$ are the type 1 polyphase components of an arbitrary transfer function $P(z)$, and $C$ is as in (16b). Then the quantity $T(z)$ defined in (4) is given by $T(z)=P(z)$.

A proof is included in the Appendix. The theorem holds no matter what $P(z)$ is, and indicates how phase distortion can be eliminated: just choose $P(z)$ to have linear phase! For notational convenience we take $P(z)$ to have zero phase so that the (real-valued) impulse response $p(n)$ is symmetric with respect to $n=0$. The passband ripples of $P(z)$ directly govern the extent of amplitude distortion of the reconstruction system. It is for this reason that we prefer to design $P(z)$ (rather that the bandpass response $B(z)$ ) to be an optimal response (say, in the equiripple sense) so that the amplitude distortion $\left|T\left(e^{j \omega}\right)\right|$ is minimized in the same sense.

1) Imposing "Mth Band" Property on $P(z)$ : Before proceeding further, another important constraint should be imposed on $P(z)$. The key observation behind the derivtion of the structure of Fig. 6 was the fact that the shifted versions $B\left(e^{j \omega} W^{k}\right)$ of the ideal response $B\left(e^{j \omega}\right)$ can be used to construct a multilevel response of the form in Fig. 4. In practice, however, we are less fortunately situated. The function $B\left(e^{j \omega}\right)$ (which is uniquely determined by $P(z)$ through (14) ) has a finite transition bandwidth, and nonzero passband and stopband ripples. The ripples eventually affect the accuracy of the multilevel approximations, but the transition bandwidth can have a more serious effect. This is demonstrated in Fig. 7. If the overlaps of $B\left(e^{j \omega}\right)$ and $B\left(e^{j \omega} W\right)$ are not carefully chosen, the sum $B\left(e^{j \omega}\right)+B\left(e^{j \omega} W\right)$ can exhibit "bumps" or "dips." As observed in [12], this effect can be overcome by forcing $B(z)$ to be an $M$ th band filter.

Recall [13] that a zero-phase transfer function $B(z)=$ $\Sigma_{n} b(n) z^{-n}$ is said to have the $M$ th band property if $b(M n)=0$ for all $n \neq 0$. This is equivalent to saying that the type 1 polyphase component $B_{0}(z)$ is a constant. We shall take this constant to be $1 / M$ for convenience. Thus the $M$ th band property is equivalent to the important property

$$
\sum_{k=0}^{M-1} B\left(z W^{k}\right)=1 .
$$

Now consider again the $\operatorname{sum} B\left(e^{j \omega}\right)+B\left(e^{j \omega} W\right)$ demonstrated in Fig. 7. If $B\left(e^{j \omega}\right)$ has sufficiently high stopband attenuation, then the shifted responses $B\left(e^{j \omega} W^{k}\right), k$ 


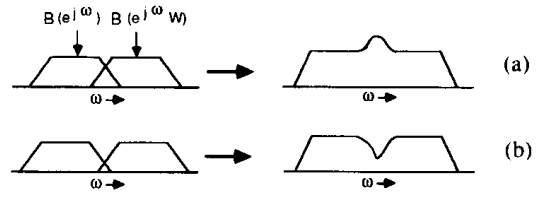

Fig. 7. The sum of $B\left(e^{j \omega}\right)$ and $B\left(e^{j \omega} W\right)$. Unless the overlap is right, the result can exhibit (a) bumps or (b) dips.

$>1$ have negligible value in the region of overlap of $B\left(e^{j \omega}\right)$ and $B\left(e^{j \omega} W\right)$ so that

$$
\sum_{k=0}^{M-1} B\left(e^{j \omega} W^{k}\right) \approx B\left(e^{j \omega}\right)+B\left(e^{j \omega} W\right) .
$$

If $B(z)$ has $M$ th band property, then the left-hand side of (20) reduces to 1 so that the right-hand side of (20) approximates unity throughout the region of overlap of the two terms. Thus no significant bumps or dips are exhibited. Next consider the summation $\Sigma_{k=0}^{M-1} \beta_{k} B\left(e^{j \omega} W^{k}\right)$ which is used to obtain a multilevel filter. Once again in the region where $B\left(e^{j \omega}\right)$ overlaps with $B\left(e^{j \omega} W\right)$, this can be approximated by

$$
\begin{aligned}
\sum_{k=0}^{1} \beta_{k} B\left(e^{j \omega} W^{k}\right) \\
\quad=\beta_{0}\left[B\left(e^{j \omega}\right)+B\left(e^{j \omega} W\right)\right]+\left(\beta_{1}-\beta_{0}\right) B\left(e^{j \omega} W\right) \\
\quad \approx \beta_{0}+\left(\beta_{1}-\beta_{0}\right) B\left(e^{j \omega} W\right)
\end{aligned}
$$

which says that as long as $B\left(e^{j \omega}\right)$ has a monotone transition band, the change from the level $\beta_{0}$ to $\beta_{1}$ is monotone or "bump free."

It is thus desirable to take $B(z)$ to be an $M$ th band filter. But in our design approach we do not have direct control over $B(z)$ because the prototype filter which we actually design is the low-pass filter $P(z)$. However, since the polyphase components of $B(z)$ and $P(z)$ are related as in (14), it is clear that $B_{0}(z)$ is constant if and only if $P_{0}(z)$ is constant. In other words, if $P(z)$ is constrained to be an $M$ th band filter, $B(z)$ inherits this property.

2) The Design Procedure: Given the quantities $M$ and $L$, the first step is to design a zero-phase FIR $M$ th band low-pass filter $P(z)$. Several techniques for FIR $M$ th band filter design are available [13], [14]. Standard specifications such as passband ripples, stopband attenuation and transition bandwidth are chosen based on the extent of amplitude distortion $\left|T\left(e^{j \omega}\right)\right|$ one is willing to tolerate in the reconstructed signal. Once $P(z)$ is designed the components $P_{l}(z)$ are known, and $G_{l}(z)$ obtained from (12). All quantities required to implement the synthesis bank of Fig. 6 are now known.

3) Advantages of the New Method Over [5]: The structure of Fig. 6 works for arbitrary $M, L$, whereas those in [5] were generated for special cases, without a unifying polyphase framework. The new method also offers direct control over the amplitude distortion $\left|T\left(e^{j \omega}\right)\right|$ as explained in the paragraph following Theorem 4. The method also has substantial computational advantages over the earlier one, as we demonstrate in Section III. Another advantage follows from an easy result induced by the $M$ th band property of $P(z)$, stated next.

Lemma 2: The $M$ th band property of $P(z)$ (i.e., the property ' $P_{0}(z)=$ constant ") implies that $G_{0}(z)$ in Fig. 6 is a constant equal to $1 / M$.

The proof is immediate from (12). By combining Lemmas 1 and 2 the reader can verify that the set of known samples, viz., $x(n M-k), 0 \leq k \leq L-1$ are not recomputed by the structure of Fig. 6; they are simply passed through the synthesis bank, so their values are not affected by rounding errors of any sort (or by the design of $P(z)$ ). Only the subset of missing samples are computed by the synthesis bank.

4) A Disadvantage of the New Method: The method does not offer direct control over the attenuation provided by the alias-component transfer functions $A_{l}(z), l>0$, even though this attenuation improves as $P(z)$ moves closer to ideal. The quantity $\left|A_{l}\left(e^{j \omega}\right)\right|$ is required to be small in the region where $\left|X\left(e^{j \omega} W^{-l}\right)\right|$ can have significant energy, but it is not clear how a prescribed specification on this attenuation can be satisfied optimally during the design of the prototype $P(z)$. A lucky exception to this is the $L=2, M=3$ case.

\section{The $L=2, M=3$ Case And A Design EXAMPLE}

We shall now demonstrate the ideas of the previous section by explicitly calculating the various matrices and functions for the special case where $L=2$ and $M=3$. In this case the vector $f^{T}(z)$ is given by

$$
\begin{aligned}
f^{T}(z) & =\frac{1}{3}\left[F_{0}(z) z^{-1} F_{1}(z)\right] \\
& =\left[G_{0}\left(z^{3}\right) z^{-1} G_{1}\left(z^{3}\right) z^{-2} G_{2}\left(z^{3}\right)\right] C .
\end{aligned}
$$

To find $\boldsymbol{C}$, notice from the definitions of $\boldsymbol{\Lambda}, \boldsymbol{R}$, and $\boldsymbol{A}$ that

$$
\begin{aligned}
\mathbf{\Lambda} & =\left[\begin{array}{lll}
1 & 0 & 0 \\
0 & W^{1 / 2} & 0 \\
0 & 0 & W
\end{array}\right], \quad \boldsymbol{R}=\left[\begin{array}{ll}
1 & 1 \\
1 & W \\
1 & W^{2}
\end{array}\right] \\
\boldsymbol{A} & =\frac{1}{W-1}\left[\begin{array}{cc}
W & -1 \\
-1 & W
\end{array}\right]
\end{aligned}
$$

where $W=e^{-j 2 \pi / 3}$. Explicit computation shows

$$
C=\left[\begin{array}{rr}
1 & 1 \\
1 & 0 \\
0 & -1
\end{array}\right]
$$

which is real and indeed has the properties advertised in Lemma 1. Substituting into (22) we arrive at

$$
\begin{aligned}
F_{0}(z) & =3\left[G_{0}\left(z^{3}\right)+z^{-1} G_{1}\left(z^{3}\right)\right] \\
z^{-1} F_{1}(z) & =3\left[G_{0}\left(z^{3}\right)-z^{-2} G_{2}\left(z^{3}\right)\right] .
\end{aligned}
$$

From (10) we verify that $\gamma_{0}=2, \gamma_{1}=1$, and $\gamma_{2}=-1$, so from (12) $G_{0}(z)=P_{0}(z) / 2, G_{1}(z)=P_{1}(z)$ and 
$G_{2}(z)=-P_{2}(z)$. Next we evaluate $A_{1}(z)$ from (3) using (24). These turn out to be

$$
\begin{aligned}
A_{0}(z)= & 2 G_{0}\left(z^{3}\right)+z^{-1} G_{1}\left(z^{3}\right)-z^{-2} G_{2}\left(z^{3}\right) \\
A_{1}(z)= & (1+W) G_{0}\left(z^{3}\right)+z^{-1} G_{1}\left(z^{3}\right)-z^{-2} W G_{2}\left(z^{3}\right) \\
A_{2}(z)= & \left(1+W^{2}\right) G_{0}\left(z^{3}\right)+z^{-1} G_{1}\left(z^{3}\right) \\
& -z^{-2} W^{2} G_{2}\left(z^{3}\right) .
\end{aligned}
$$

Notice that by definition, $A_{0}(z)$ is the distortion function $T(z)$. We know from Theorem 4 that this is supposed to equal $P(z)$. This indeed is the case as can be verified by replacing $G_{l}(z)$ in (25a) in terms of $P_{l}(z)$.

Imposing the third band constraint on $P(z)$ results in $P_{0}(z)=2 / 3$ so that $P(z)=\frac{2}{3}+z^{-1} P_{1}(z)+z^{-2} P_{2}(z)$. Assuming further that $P(z)$ is a real-coefficient zero-phase filter, we have $P(z)=P\left(z^{-1}\right)$. From this it follows that $P_{2}(z)=z P_{1}\left(z^{-1}\right)$. These relations among $P_{1}(z)$ induce relations among $G_{l}(z)$ 's in view of (12). Summarizing, we have

$$
\begin{array}{ll}
P_{0}(z)=\frac{2}{3}, & P_{2}(z)=z P_{1}\left(z^{-1}\right) \\
G_{0}(z)=\frac{1}{3}, & G_{2}(z)=-z G_{1}\left(z^{-1}\right) .
\end{array}
$$

By using these in (25) and simplifying, we obtain the relations

$$
\begin{aligned}
& A_{2}(z)=W\left[A_{0}(z W)-1\right] \\
& A_{1}(z)=W A_{2}(z W)
\end{aligned}
$$

so that $\left|A_{1}\left(e^{j \omega}\right)\right|=\left|A_{2}\left(e^{-j \omega}\right)\right|$. Using the fact that $A_{0}(z W)$ has zero phase, we give qualitative sketches of $\left|A_{l}\left(e^{j \omega}\right)\right|$ in Fig. 8 for $l=0,1,2$. It is clear in this case that the attenuations provided by $A_{1}(z)$ and $A_{2}(z)$ are directly related to the passband ripple of $A_{0}(z)$ (i.e., of the prototype $P(z))$. So the passband ripple of $P(z)$ can be used to control the aliasing as well as amplitude distortion effects. Unlike in the arbitrary $M, L$ case, we can therefore control both aliasing and amplitude distortion directly by design of $P(z)$.

\section{A. A Design Example}

We shall assume that $x(n)$ is band limited to $|\omega| \leq \omega_{p}$ where $\omega_{p}<2 \pi / 3$. The gap $\Delta \omega \triangleq 2 \pi / 3-\omega_{p}$ helps us to use filters with nonzero transition bands. Since the distortion function $T(z)=P(z)$, we shall choose $P(z)$ to be low pass with passband edge at $\omega_{p}$. Our specifications for $P(z)$ in this example will be 1) $\omega_{p}=2 \pi / 3-0.034 \pi$ so that $\Delta \omega=0.034 \pi$, and 2) peak passband ripple 0.001 . Because of the third band constraint on $P(z)$, its stopband features cannot be independently specified [13]. Several standard methods exist for design of third band filters [13], [14]; here we use the method in [17], which is a Remeztype exchange algorithm, providing equiripple passband. The order of $P(z)$ for the above specifications turns out to be 94 . From (27), the stopband attenuations provided by $A_{1}(z)$ and $A_{2}(z)$ are $60 \mathrm{~dB}$. The reader can verify that (a)

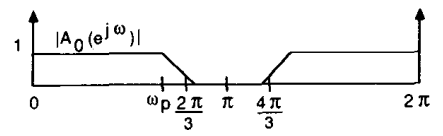

(b)

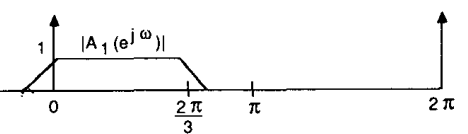

(c)

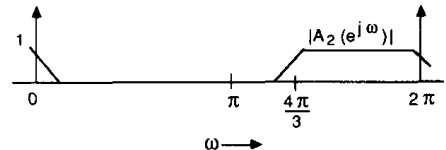

Fig. 8. Qualitative plots of $\left|A_{1}\left(e^{j \omega}\right)\right|$ for $L=2, M=3$ case. (a) $l=0$, (b) $l=1$, (c) $l=2$.

the support of $X\left(e^{j \omega}\right)$ coincides precisely with the passband of $P(z)$ and the supports of $X\left(z W^{-1}\right)$ and $X\left(z W^{-2}\right)$ coincide exactly with the stopbands of $A_{1}(z)$ and $A_{2}(z)$. Figs. 9(a) and (b) show the plots of $\left|P\left(e^{j \omega}\right)\right|$ and $\left|A_{2}\left(e^{j \omega}\right)\right|$. Using the same test signal $x(n)$ as in [5], we obtain the plot $\left|\hat{X}\left(e^{j \omega}\right)\right|$ shown in Fig. 9(c) for the reconstructed signal $\hat{x}(n)$. This plot agrees well with the plot of $\left|X\left(e^{j \omega}\right)\right|$ shown in [5]. Since $P(z)$ has linear phase, $\hat{x}(n)$ is automatically free from phase distortion.

In order to compare this method with the one in [5], we have to design the low-pass filter $G_{L}(z)$ and the Hilbert transformer $G_{H}(z)$ in [5, fig. 17] such that $T(z), A_{1}(z)$, and $A_{2}(z)$ meet the same specifications as above. The specifications of importance are: 1) passband width and ripple of $T(z), 2)$ stopband regions and stopband attenuations of $A_{1}(z)$ and $A_{2}(z)$. The reader can verify that $T(z)$ and $A_{1}(z)$ are related to $G_{L}(z)$ and $G_{H}(z)$ as follows: $T(z)=z^{-1} G_{L}(z), A_{1}(z)=\left[z^{-1} G_{L}(z) / 4\right][1-$ $\left.j \sqrt{3}-(\sqrt{3}+j) G_{H}(z)\right]$. Also $A_{2}(z)$ satisfies $\left|A_{2}\left(e^{j \omega}\right)\right|$ $=\left|A_{1}\left(e^{-j \omega}\right)\right|$. Our aim now is to find the specifications on $G_{L}(z)$ and $G_{H}(z)$ so that $T(z), A_{1}(z)$ and $A_{2}(z)$ will have same performance as in the new method. Let $\phi_{p}, \phi_{S}$, $\epsilon_{p}$, and $\epsilon_{S}$ denote the passband edge, stopband edge, peak passband ripple, and peak stopband ripple of $G_{L}(z)$. Let $\theta_{p}$ and $\theta_{S}$ be the band edges of $G_{H}(z)$ (with same meaning as in [5, fig. 18(b)]) and let $\delta$ denote its peak passband ripple. By sketching the functions $\left|T\left(e^{j \omega}\right)\right|,\left|A_{1}\left(e^{j \omega}\right)\right|$, and $\left|A_{2}\left(e^{j \omega}\right)\right|$, the reader can verify that this method meets the same requirements as in the previous paragraph if we choose $\phi_{p}=2 \pi / 3-\Delta \omega, \phi_{S}=2 \pi / 3+\Delta \omega, \epsilon_{p}=$ $\epsilon_{S}=0.001, \theta_{p}=\Delta \omega$, and $\delta=0.002$. We choose $\theta_{S}=$ $\pi-\theta_{p}$ for reason described in [5]. These specifications can be met by a linear phase FIR $G_{L}(z)$ of order 96 , and a linear phase Hilbert transformer $G_{H}(z)$ or order 98 . The number of multipliers to implement these are: 49 for $G_{L}(z)$ and only 25 for $G_{H}(z)$ (due to symmetry of $\left|G_{H}\left(e^{j \omega}\right)\right|$ with respect to $\left.\pi / 2[15]\right)$. So this requires a total of 76 multipliers (counting $s$ and $1-c$ in [5, fig. 17]).

Finally, we shall compare these methods with the more traditional way [6] to fractionally compress a signal $x(n)$ band limited as above. This is shown in Fig. 10. Here 


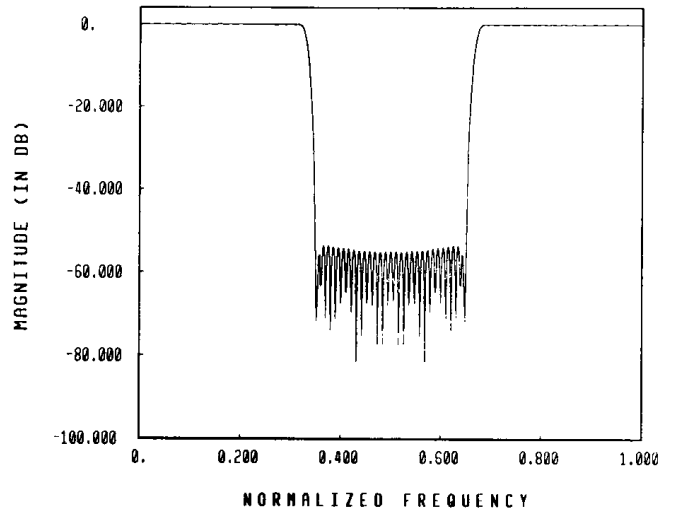

(a)

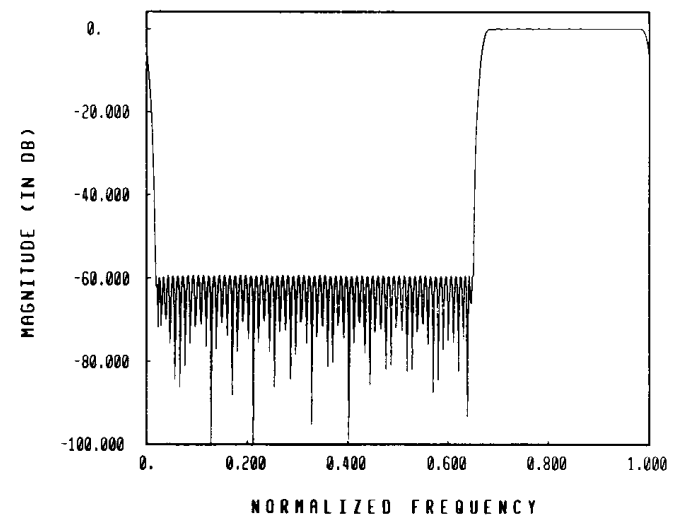

(b)

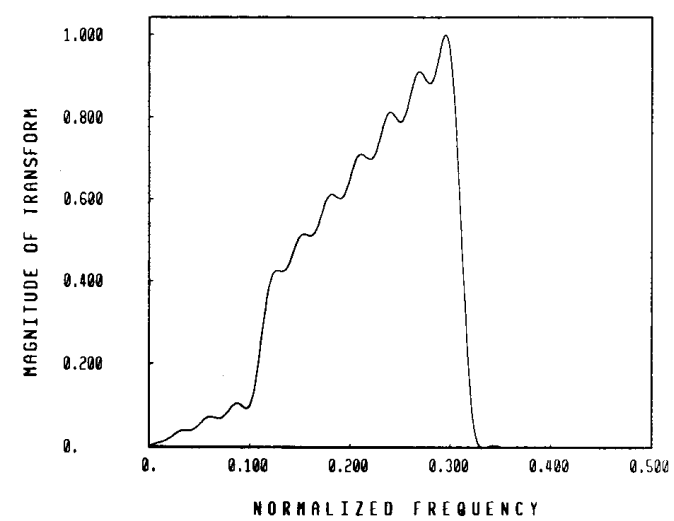

(c)

Fig. 9. Plots of magnitudes of (a) $P\left(e^{j \omega}\right)=T\left(e^{j \omega}\right)$, (b) $A_{2}\left(e^{j \omega}\right)$, and (c) $\hat{X}\left(e^{j \omega}\right)$, for the design example.

$y(n)$ is the compressed signal and $v(n)$ is the reconstructed signal at the original rate (i.e., the rate of $x(n)$ ). To facilitate our discussions, we shall assign the following names to the three methods:

Method I: the one shown in Fig. 10 (earliest method [6]).

Method II: the one reported in [5, fig. 17] (recent method).

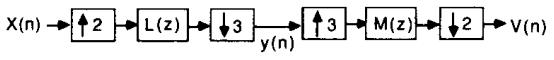

Fig. 10. The most well-known method for fractional decimation and reconstruction.

Method III: the one reported in this paper.

It remains to estimate the cost of method I now. By sketching the various internal waveforms in Fig. 10 one can see that $L(z)$ can be designed to be a low-pass halfband filter [13] with a large transition bandwidth $(=\pi / 3$ $+\Delta \omega)$. So its cost is very low, and we can afford to make its in-band ripples sufficiently small so that the accuracy of the reconstructed signal $v(n)$ is determined primarily by $M(z)$. In order to meet the same requirements on this accuracy as methods II and III, the following specifications were used for $L(z)$ and $M(z)$. 1) $L(z)$ : half-band linear-phase FIR with peak passband and stopband ripples $=0.0001$, and transition bandwidth $=\pi / 3+\Delta \omega .2$ ) $M(z)$ : third-band linear-phase FIR with peak passband and stopband ripples $=0.001$ and transition bandwidth $=$ $\Delta \omega$. The orders of these filters turn out to be 26 and 190 , respectively. By exploiting the half-band and third band properties of $L(z)$ and $M(z)$, respectively, (and also their linear-phase property) we can implement these with 7 and 64 multipliers, respectively, i.e., a total of 71 multipliers. The new method (method III) requires fewer than half as many multipliers as either of the earlier methods.

\section{B. Comparison Based on Multiplications Per Unit Time}

It is often more appropriate to perform the complexitycomparison based on the number of multiplications per unit time (defined as the interval between successive samples of the original signal $x(n))$. For method I, the filters $L(z)$ and $M(z)$ can be implemented very efficiently by simultaneous exploitation of the presence of decimators and interpolators as described in [12], [16]. If this is done, one can verify that $L(z)$ requires only 3 MPU's and $M(z)$ requires 22 so the total is 25 MPU's. For method II, it is not possible to rearrange the flowgraph of $[5$, fig. 17$]$ in order to exploit the presence of decimators and interpolators so that the number of MPU's remains equal to 76 . For method III we can implement the reconstruction system as in Fig. 11, requiring a total of only 11 MPU's. Table I summarizes the comparison of the three methods. The advantage offered by method III is obvious.

\section{Nonconsecutive Sampling and Multiband Signals}

In Section II we retained $L$ consecutive samples $x$ ( $n M$ $-k), 0 \leq k \leq L-1$ from each length- $M$ segment of $x(n)$. We now consider two generalizations: first, we retain $x\left(n M-n_{k}\right), 0 \leq k \leq L-1$ where $n_{k}$ are not necessarily consecutive, but satisfy $0 \leq n_{0}<n_{1}<\cdots<$ $n_{L-1}<M$. Second, we do not restrict $x(n)$ to be low pass but consider it to be a multiband signal. Such a signal is one for which $X\left(e^{j \omega}\right)$ is nonzero in a subset of $L$ regions out of the $M$ regions shown in Fig. 12. Let $R_{l_{q}}, 0 \leq q \leq$ 


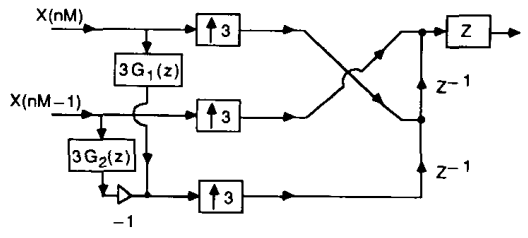

Fig. 11. Rearranging the reconstruction system for the design example (method III)

TABLE I

Comparison of the Three Reconstruction Methods in Terms of Number OF MULTiPlications

\begin{tabular}{|c|c|c|c|}
\hline Mlathod & Filter Orders & $\begin{array}{l}\text { Total } \\
\text { Number } \\
\text { of } \\
\text { Multipliers }\end{array}$ & $\begin{array}{l}\text { Total } \\
\text { Number } \\
\text { of } \\
\text { MPUS } \\
\end{array}$ \\
\hline 1 & $\begin{array}{l}L(z): 26 \\
M(z): 190\end{array}$ & 71 & 25 \\
\hline ॥ & $\begin{array}{l}C_{1}(z): 96 \\
\operatorname{Cin}_{1}(z): 98\end{array}$ & 76 & 76 \\
\hline III & $P(z): 94$ & 32 & 11 \\
\hline
\end{tabular}

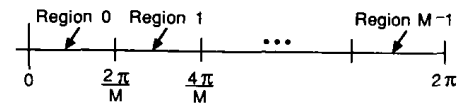

Fig. 12. Definition of the $M$ regions for the multiband case.

$L-1$ denote these $L$ regions, let $\&$ be the set $\left\{l_{0}, l_{1}\right.$, $\left.\cdots, l_{L-1}\right\}$, and let $B$ be the union of these $L$ regions (i.e., $B$ is the support of $X\left(e^{j \omega}\right)$ ). Since $M$ and $\mathscr{L}$ completely characterize the frequency bands, we shall say that $x(n)$ is an $(M, \mathscr{L})-B L$ signal (extension of the $\sigma-B L$ jargon!). Since the total spectral occupancy of $x(n)$ is only $2 \pi L / M$, it is possible to compress the signal by $M / L$. Suppose we compress $x(n)$ by nonuniform nonconsecutive decimation, i.e., by retaining $x\left(n M-n_{k}\right), 0 \leq n_{k}$ $\leq L-1$. Can we still recover $x(n)$ by use of a synthesis bank $\left\{F_{k}(z)\right\}$ ?

The answer is yes, provided the set $\left\{n_{k}\right\}$ is chosen properly. In particular, if $\left\{n_{k}\right\}$ are consecutive, such reconstruction is always possible. We shall now quantitatively state these and related results. The developments are analogous to those in Section II though notationally more tedious. Proofs can be found in [17].

The nonconsecutive nonuniform decimation can be represented as in Fig. 1 except that the delay elements are now $z^{-n_{k}}, 0 \leq k \leq L-1$. Once again $\hat{x}(n)$ suffers from the same set of distortions as in Section II.

Define $f^{\prime}(z)=(1 / M)\left[z^{-n_{0}} F_{0}(z) \quad \cdots\right.$ $\left.z^{-n L-1} F_{L-1}(z)\right]^{T}, V^{\prime}$ to be the $L \times L$ matrix with elements $V_{m k}^{\prime}=W^{n k l_{m}}$, and $e_{q}$ to be the $q$ th unit vector. If $\left\{F_{k}(z)\right\}$ is the set of $L$ synthesis filters resulting in perfect reconstruction (i.e., $\hat{x}(n)=x(n)$ ), then $\boldsymbol{V}^{\prime} \boldsymbol{E}_{q}^{-1} \boldsymbol{f}^{\prime}\left(\boldsymbol{e}^{j \omega}\right)=\boldsymbol{e}_{q}$ in region $R_{l_{q}}$, where $E_{q}=\operatorname{diag}\left[W^{l_{q} n 0}, \cdots, W^{l_{q} n_{L}-1}\right]$. So

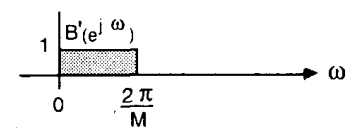

Fig. 13. Prototype zero-phase filter for multiband case.

we can solve for $f^{\prime}\left(e^{j \omega}\right)$ provided that $V^{\prime}$ is nonsingular. This, in general, is not guaranteed for arbitrary $\left\{n_{k}\right\}$ and $\left\{l_{m}\right\}$. In the special case where $n_{k}$ are consecutive integers or when $l_{m}$ are consecutive integers, $V^{\prime}$ does become nonsingular because it (or its transpose) is then a Vandermonde matrix with distinct columns. Assuming that $V^{\prime}$ is nonsingular, the solution vector $\boldsymbol{f}^{\prime}$ is such that each $z^{-n_{k}} F_{k}(z)$ is again a multilevel filter, with $F_{k}\left(e^{j \omega}\right)=0$ for $\omega \notin B$. So we can again obtain all these ideal filters in terms of a prototype zero-phase $M$ th band filter with response $B^{\prime}\left(e^{j \omega}\right)$ shown in Fig. 13. Equivalently, we can use the polyphase components of a zero-phase $M$ th band prototype $P^{\prime}\left(e^{j \omega}\right)$ whose response equals unity for $\omega \in \mathbb{B}$ and zero otherwise. The advantage of using $P^{\prime}\left(e^{j \omega}\right)$ is that, when the ideal filter is replaced with a practical approximation, the distortion function $T(z)$ is equal to $P^{\prime}(z)$ so that we have direct control over the specifications of $T(z)$ while designing $P^{\prime}(z)$. The resulting synthesis bank can once again be expressed as in Fig. 6 with some obvious modification such as using primed versions, etc. The matrix $C$ is also appropriately replaced. This matrix $C$ completely summarizes the nonuniform decimation pattern $\left\{n_{k}\right\}$ and the multiband pattern $\left\{l_{m}\right\}$. The expression for $f^{\prime}(z)$ in terms of $P_{l}^{\prime}(z)$ (which are the polyphase components of $P^{\prime}(z)$ ) contains terms of the form $P_{n}^{\prime}(z) / q_{n}^{\prime}$ where $q_{n}^{\prime}=\Sigma_{i=0}^{L-1} W^{-n l_{i}}$. Unlike $q_{l}$ defined in (10), $q_{n}^{\prime}$ can be zero, so the use of $P^{\prime}(z)$ as a prototype may not be feasible. However, it is always possible to use $B^{\prime}(z)$ in order to generate all the $L$ multilevel filters $z^{-n_{k}} F_{k}(z)$. Once again, if $l_{m}, 0 \leq m \leq L-1$, are consecutive then $q_{i}^{\prime}$ are guaranteed to be nonzero.

The usefulness of these results on generalized nonuniform decimation of multiband signals will now be demonstrated by a simple example. Let $L=2, M=4$, and let $X\left(e^{j \omega}\right)$ be as in Fig. 14(a). Here $l_{0}=1, l_{1}=3$. Our aim is to keep two out of every four samples from $x(n)$ in such a way that $x(n)$ can eventually be reconstructed. If we choose $n_{0}=0, n_{1}=2$ (uniform decimation by two) then $V^{\prime}=\left[\begin{array}{ll}1 & W^{2} \\ 1 & W^{2}\end{array}\right]$, which is singular. So the reconstruction scheme will fail. On the other hand, if we choose $n_{0}=0$ and $n_{1}=1$ (nonuniform decimation) then $V^{\prime}=\left[\begin{array}{ll}1 & W \\ 1 & W^{3}\end{array}\right]$ which is nonsingular so that the reconstruction can be carried out. Note, incidentally, that unlike in Section II (lowpass case), it makes sense to have nontrivial common factors between $L$ and $M$ here. The reason why uniform decimation fails in this example can also be seen in the frequency domain: When $x(n)$ is decimated uniformly by two, the result has Fourier transform $\left[X\left(e^{j(\omega / 2}\right)+\right.$ $X\left(e^{j(\omega-2 \pi) / 2}\right] / 2$ and the two components overlap (Fig. 14(b)). So $x(n)$ cannot be recovered. 

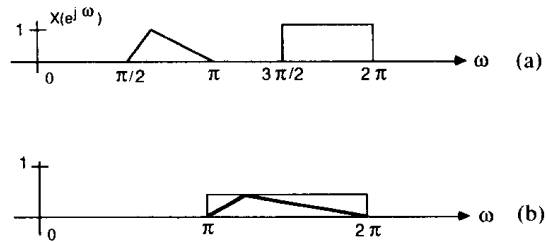

Fig. 14. Example of (a) a multiband $X\left(e^{j \omega}\right)$ and (b) the uniformly decimated version.

\section{Multidimensional Extensions}

The results of the previous sections easily extend to the multidimensional case. We shall briefly summarize the results here using, for convenience, the $2 \mathrm{D}$ case. Some details can be found in [17]. The extensions are based on integer sublattices and cosets [18]-[20] which are related to the notions of generalized (non rectangular) decimators and generalized polyphase components [20].

\section{A. Review from Integer Lattice Theory}

The set $Z^{2}$ of all $2 \times 1$ integer vectors is called the $2 D$ integer lattice. Any $2 \times 2$ integer matrix $D$ with det $[D]$ $=M>0$ generates a sublattice $\Lambda_{D}$ of $Z^{2}$, which is the set of all integer vectors of the form $D n, n \in Z^{2}$. A coset $\boldsymbol{\Lambda}_{D}\left(\boldsymbol{n}_{i}\right)$ of $\boldsymbol{\Lambda}_{D}$, generated by any integer vector $\boldsymbol{n}_{i}$ is the set of all integer vectors of the form $n_{i}+D n, n \in Z^{2}$. The vector $n_{i}$ is also loosely called a "shift-vector." An important result in lattice theory says that for any integer matrix $D$ with determinant $M>0$, there exists a set of $M$ shift vectors $\left\{\boldsymbol{n}_{0}, \boldsymbol{n}_{1}, \cdots, \boldsymbol{n}_{M-1}\right\}$ such that any $\boldsymbol{n} \in \mathcal{Z}^{2}$ can be expressed as $n=n_{i}+D m$ for some $m \in Z^{2}$. Thus $Z^{2}$ is a union of $M$ cosets. Note the analogy to the $1 \mathrm{D}$ case: any integer $n \in \mathcal{Z}$ can be represented as $n_{i}+M m$, $0 \leq n_{i} \leq M-1, m \in \mathcal{Z}$. This analogy can be used to define polyphase decomposition for multidimensional signals.

Now let $\boldsymbol{E} \triangleq 2 \pi D^{-T}$. Let $\beta$ denote the set of vectors of the form $E \boldsymbol{a}, \boldsymbol{a} \in[0,1)^{2}$. Then there exists a set of $M$ integer vectors $\left\{\boldsymbol{m}_{0}, \boldsymbol{m}_{1}, \cdots, \boldsymbol{m}_{M-1}\right\}$ such that any $2 \mathrm{D}$ frequency $w \in[0,2 \pi)^{2}$ can be expressed as $w=w_{0}+$ $\boldsymbol{E} \boldsymbol{m}_{i}$ for some $\boldsymbol{w}_{0} \in \mathcal{P}$, and some $\boldsymbol{m}_{i}$ in the above set. For a fixed $\boldsymbol{m}_{i}$ denote $R_{m_{i}}$ to be the set of all $\boldsymbol{w}$ that can be expressed as $\boldsymbol{w}_{0}+\boldsymbol{E} \boldsymbol{m}_{i}, \boldsymbol{w}_{0} \in \mathcal{P}$. Then $[0,2 \pi)^{2}$ is the union of the $M$ regions $R_{m_{i}}$.

\section{B. Application to Nonuniform Decimation}

Any integer matrix $\boldsymbol{D}$ with determinant $M>0$ can be used to partition the integer set $\mathfrak{Z}^{2}$ into $M$ cosets determined by the $M$ integers $n_{i}$. From $D$ we can find a corresponding partitioning of the frequency domain $[0,2 \pi)^{2}$ into $M$ regions, determined by the $M$ integer vectors $\boldsymbol{m}_{i}$. We say that the 2D signal $x(\boldsymbol{n})$ is multiband bandlimited if $X(\boldsymbol{w})$ is nonzero only in $L$ out of these $M$ regions, $L<$ $M$. Let the $L$ regions be $R_{m_{i}}, 0 \leq i \leq L-1$. We should now appropriately define a nonuniform decimation scheme which keeps $L$ out of $M$ samples. Suppose we retain the samples $x\left(D n+n_{i}\right)$ with $0 \leq i \leq L-1$. In other words, only the samples belonging to the first $L$ of the $M$ cosets are retained. Is it possible to reconstruct $x(n)$ from these samples by using a $2 \mathrm{D}$ version of the synthesis bank? The answer again is in the affirmative, provided the coset of retained samples is carefully chosen (elaborated below). The decimation and reconstruction process can be formulated in a way analogous to that in Fig. 1. Thus, we can use the system shown in [20, fig. 1], with a subset of analysis filters taken to be $2 \mathrm{D}$ advance operators and the others to be zero. The corresponding set of $L$ synthesis filters which gives rise to perfect recovery can again be obtained by solving a set of $L$ equations. There are $L$ sets of such equations, one for each of the $L$ regions $R_{m_{i}}$. All these sets of equations involve the inversion of a fixed $L$ $\times L$ matrix, whose entries are given by $e^{-j 2 \pi m_{i}^{I} D^{-1} n_{k}}, 0 \leq$ $i, k \leq L-1$. So the reconstruction can be carried out if this matrix is nonsingular. For the special case where the shift vectors $\boldsymbol{n}_{k}$ take the form $\boldsymbol{n}_{k}=k \boldsymbol{m}, 0 \leq k \leq M-1$, $\boldsymbol{m} \neq \mathbf{0}$, this matrix is guaranteed to be nonsingular provided the retained samples are $x(D n+k m)$ for $L$ consecutive values of $k$. This is true regardless of the subset $\boldsymbol{m}_{i}$ which represents the $L$ regions of support of $X(w)$.

\section{APPENDIX}

Proof of Lemma 1: We shall first prove the fourth property, i.e., that $C$ defined as in (16b) is real. This result follows from an important property satisfied by the columns $\boldsymbol{u}_{i}$ of the matrix $U \triangleq V^{-1}$, viz.,

$$
D_{i} u_{i}=D_{L-1-i}^{*} \boldsymbol{u}_{L-1-i}^{*}, \quad 0 \leq i \leq L-1 .
$$

To prove this property of $\boldsymbol{u}_{i}^{\prime} s$, note that $\boldsymbol{V} \boldsymbol{u}_{i}=\boldsymbol{e}_{i}$ where $\boldsymbol{e}_{i}$ is the $i$ th unit vector. So $J V u_{i}=e_{L-1-i}$ where $J$ is the flipping matrix defined in Section I. But from the definition of $\boldsymbol{V}$ we can verify $\boldsymbol{J} \boldsymbol{V}=\boldsymbol{V}^{*} \boldsymbol{D}_{L-1}$ so that " $\boldsymbol{J} \boldsymbol{V} \boldsymbol{u}_{i}=$ $\boldsymbol{e}_{L-1-i}$ " implies " $\boldsymbol{V} * \boldsymbol{D}_{L-1} \boldsymbol{u}_{i}=\boldsymbol{e}_{L-1-i}$ " which implies

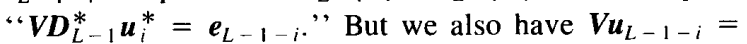
$\boldsymbol{e}_{L-1-i}$ which proves that $\boldsymbol{u}_{L-1-i}=D_{L-1}^{*} \boldsymbol{u}_{i}^{*}$ implying (A.1) indeed. Now $\boldsymbol{A}$ is given by (17) so that

$$
\boldsymbol{J A}=\boldsymbol{A}^{*}
$$

in view of (A.1). From the definition of $\boldsymbol{R}$ it also follows that

$$
\boldsymbol{R} \boldsymbol{J}=\boldsymbol{\Lambda}^{2} \boldsymbol{R}^{*}
$$

By using (A.2) and (A.3) we obtain $\boldsymbol{R} \boldsymbol{A}^{*}=\boldsymbol{R} \boldsymbol{J} \boldsymbol{J} \boldsymbol{A}^{*}=$ $\Lambda^{2} R^{*} \boldsymbol{A}$, which proves that $\Lambda^{*} \boldsymbol{R} \boldsymbol{A}^{*}=\boldsymbol{\Lambda} \boldsymbol{R}^{*} \boldsymbol{A}$, i.e., that $\boldsymbol{C}$ is real. Now consider proving Properties 1-3. This is equivalent to proving that $\boldsymbol{R}^{*} \boldsymbol{A}$ has these same properties. If we use the following facts: 1) $V$ is the upper $L \times L$ submatrix of the $M \times L$ matrix $R, 2) V$ is the inverse of $\boldsymbol{U}$, and 3) the rows of $\boldsymbol{A}$ in (17) are determined by the columns $\boldsymbol{u}_{i}$ of $\boldsymbol{U}$, we can easily verify that $R^{*} \boldsymbol{A}$ does satisfy these properties. This completes the proof of Lemma 1.

Proof of Theorem 4: Recall that $T(z)$ is defined as in (4), so $T(z)=f^{T}(z) 1$ where $1 \triangleq[11 \cdots 1]^{T}$. From 
(16a), it thus follows that $T(z)=g^{T}(z) \Lambda \boldsymbol{R}^{*} \boldsymbol{A} \mathbf{1}$. From the definition of $A$ and from the fact that $U V=V U=I$, we have $\boldsymbol{A} 1=1$ so that $R^{*} \boldsymbol{A} 1=R^{*} 1$. But from (10) we have $\boldsymbol{R}^{* 1}=\left[q_{0} q_{1} \cdots q_{M-1}\right]^{T}$. Using this with (10) we get $T(z)=\boldsymbol{g}^{T}(z) \boldsymbol{v}$ where $\boldsymbol{v}=\left[\begin{array}{llll}\gamma_{0} & \gamma_{1} & \cdots & \gamma_{M-1}\end{array}\right]^{T}$. Using (12) this gives $T(z)=P(z)$.

\section{REFERENCES}

[1] C. E. Shannon, "Communications in the presence of noise," Proc IRE, vol. 37, pp. 10-21, Jan. 1949

[2] A. Papoulis, "Generalized sampling expansion," IEEE Trans. Circuits Syst., pp. 652-654, Nov. 1977.

[3] J. L. Brown, Jr., "Multichannel sampling of low-pass signals," IEEE Trans. Circuits Syst., vol. 28, pp. 101-106, Feb. 1981.

[4] A. J. Jerri, "The Shannon sampling theorem-its various extensions and applications: A tutorial review," Proc. IEEE, pp. 1565-1596, Nov. 1977.

[5] P. P. Vaidyanathan and V. C. Liu, "Classical sampling theorems in the context of multirate and polyphase digital filter bank structures,' IEEE Trans. Acoust., Speech, Signal Processing, vol. 36, pp. 1480 1495. Sept. 1988

[6] R. E. Crochiere and L. R. Rabiner, Multirate Digital Signal Processing. Englewood Cliffs, NJ: Prentice-Hall, 1983.

[7] M. J. T. Smith and T. P. Barnwell, III, "A new filter-bank theory for time-frequency representation," IEEE Trans. Acoust., Speech, Signal Processing, vol. 35, pp. 314-327, Mar. 1987.

[8] P. P. Vaidyanathan, "Theory and design of $M$-channel maximally decimated quadrature mirror filters with arbitrary $M$, having perfect reconstruction property," IEEE Trans. Acoust., Speech. Signal Processing, vol. 35, pp. 476-492, Apr. 1987.

[9] M. Vetterli, "A theory of multirate filter banks," IEEE Trans. Acoust., Speech, Signal Processing, vol. 35, pp. 356-372, Mar. 1987.

[10] P. P. Vaidyanathan, "Quadrature mirror filter banks, $M$-band extensions and perfect-reconstruction techniques," IEEE ASSP Mag., vol. 4, pp. 4-20, July 1987 .

[11] M. Bellanger, G. Bonnerot, and M. Coudreuse, "Digital filtering by polyphase network: Application to sample rate alteration and filter banks," IEEE Trans. Acoust., Speech, Signal Processing, vol. 24, pp. 109-114, Apr. 1976

[12] P. P. Vaidyanathan, "Multirate digital filters, filter banks, polyphase networks, and applications: A tutorial," Proc. IEEE, to be published.

[13] F. Mintzer, "On half-band, third-band, and Nth band FIR filters and their design,' IEEE Trans. Acoust., Speech, Signal Processing, vol. 30, pp. 734-738, Oct. 1982 .

[14] P. P. Vaidyanathan and T. Q. Nguyen, "Eigenfilters: A new approach to least squares FIR filter design and applications including Nyquist filters," IEEE Trans. Circuits Syst., vol. 34, pp. 11-23, Jan. 1987.

[15] L. R. Rabiner and B. Gold, Theory and Application of Digital Signal Processing. Englewood Cliffs, NJ: Prentice-Hall, 1975.

[16] C.-C. Hsiao, "Polyphase filter matrix for rational sampling rate conversions," in Proc. IEEE Int. Conf. ASSP (Dallas, TX), Apr. 1987 pp. $2173-2176$

[17] V. C. Liu, "One- and two-dimensional digital multirate systems with applications in subsampling and band-limited signal reconstruction,' doctoral dissertation, Dep. Elec. Eng., California Institute Technology, Pasadena, CA, 1989.
[18] E. Dubois, "The sampling and reconstruction of time-varying imagery with application in video systems," Proc. IEEE, vol. 73, pp. 502-522, Apr. 1985

[19] D. E. Dudgeon and R. M. Mersereau, Multidimensional Digital Sig. nal Processing. Englewood Cliffs, NJ: Prentice-Hall, 1984.

[20] E. Viscito and J. Allebach, "Design of perfect reconstruction multidimensional filter banks using cascaded Smith form matrices," in Proc. IEEE Int. Symp. Circuits Syst. (Espoo, Finland), June 1988, pp. 831-834.

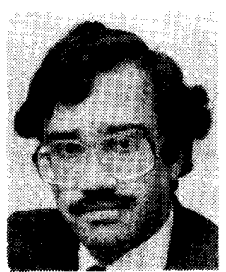

P. P. Vaidyanathan (S'80-M'83-SM'88) was born in Calcutta, India, on October 16, 1954. He received the B.Sc. (Hons.) degree in physics, and the B.Tech. and M.Tech. degrees in radiophysics and electronics from the University of Calcutta, India, in 1974, 1977, and 1979, respectively, and the Ph.D. degree in electrical and computer engineering from the University of California, Santa Barbara, in 1982.

He was a postdoctoral fellow at the University of California, Santa Barbara, from September 1982 to February 1983. In March 1983, he joined the California Institute of Technology, Pasadena, as an Assistant Professor of Electrical Engineering, and is currently an Associate Professor in the same Department. His main research interests are in digital signal processing, multirate filter bank systems, filter design, adaptive filtering, and multivariable system theory

Dr. Vaidyanathan served as the Vice-Chairman of the Technical Program Committee for the 1983 IEEE International Symposium on Circuits and Systems and as an Associate Editor for the IEEE TRANSACTIONS ON CIRCUITS AND SYSTEMS, for the period 1985 to 1987 . He received the award for Excellence in Teaching from the California Institute of Technology, for the year 1983-1984. He was also a recipient of NSF's Presidential Young Investigator Award, starting from 1986. In 1989 he received the IEEE ASSP Senior Award for his paper on multirate perfect-reconstruction filter banks. In 1990 he received the S. K. Mitra Memorial Award from the Institute of Electronics and Telecommunications Engineers, India, for his paper (with coauthor V. Liu) in the IETE Journal.

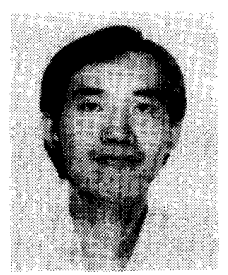

Vincent C. Liu (S'85-M'89) was born in Hong Kong in 1961. He received the B.E. degree in electrical engineering from the State University of New York at Stony Brook in 1984 , the M.S. degree in electrical engineering from the California Institute of Technology, Pasadena, in 1985, and the $\mathrm{Ph} . \mathrm{D}$. degree in electrical engineering from the California Institute of Technology in 1989.

$\mathrm{He}$ is now a Senior Engineer with the Videocipher Division of the General Instrument Corporation, San Diego, CA. His research interests include digital signal processing, two-dimensional systems, and multirate filter bank applications.

Dr. Liu was a recipient of the Schlumberger Fellowship, and is a member of Tau Beta $\mathrm{Pi}$. 